\title{
Análise da Variância - dois casos estudados
}

A análise da variância a um critério de classificação ou a um factor é uma técnica estatística correntemente utilizada para comparar as médias de várias populações supostas normais e com a mesma variância, a partir de amostras aleatórias, simples e independentes. Tem essencialmente por princípio dividir a variância total em duas quantidades: o quadrado médio factorial ou entre amostras - definida a partir dos desvios entre as médias das diferentes amostras e a média geral - e o quadrado médio residual ou dentro das amostras que é função dos desvios existentes entre os valores observados e a média das amostras correspondentes. As diferenças entre médias são testadas pelo cálculo do índice estatístico $\mathrm{F}$ que compara a variabilidade de valores entre grupos com a variabilidade entre valores individuais dentro do grupo.

\section{Caso estudado I}

O estudo tem por finalidade analisar o comportamento de um grupo de estudantes ao longo do trabalho de laboratório, TL, estabelecido para o curso de Química Geral desenvolvido em regime de acompanhamento de ritmo próprio [1]. A Tabela 1 refere as classificações médias atribuídas ao grupo nos meses de Outubro, Novembro e Dezembro. Os valores de F obtidos por execução do programa “'Análise da variância a um factor" estão referidos no Quadro I. A comparação dos valores F observados com os valores teóricos [2] permite-nos as seguintes conclusões: o valor $(3,67)$ de F para as amostras é menor do que o valor teórico $(3,72)$ para 2 e 14 graus de liberdade, ao nível 0,05 , o que conduz à aceitação da hipótese nula e, consequentemente, à conclusão de que não há diferenças nas classificações médias ao longo do período referido. Contudo, o valor $\mathrm{F}(5,26)$ para os estudantes é superior ao valor teórico $(3,65)$ para 14 graus de liberdade, ao nível 0,05 , pelo que temos de rejeitar a hipótese nula e concluir que há diferenças significativas entre os estudantes, quando considerados dentro de cada uma das amostras.

A análise da variância a um critério de classificação pode ser generalizada a dois critérios de classificação. Neste caso a soma total dos quadrados dos desvios divide-se em quatro componentes: duas são as somas factoriais, a terceira ligada à interacção dos dois factores controlados e a quarta é uma soma residual.
TABELA 1

Classificação média dos estudantes (amostra: $N=15$ estudantes do C.E. do ano lectivo de 1986/87)

\begin{tabular}{|c|c|c|c|}
\hline \multirow[t]{2}{*}{ Estudante } & \multicolumn{3}{|c|}{ Classificações médias } \\
\hline & Outubro & Novembro & Dezembro \\
\hline E1 & 55 & 65 & 50 \\
\hline E2 & 60 & 65 & 60 \\
\hline E3 & 75 & 65 & 60 \\
\hline $\mathrm{E} 4$ & 65 & 70 & 75 \\
\hline E5 & 45 & 65 & 70 \\
\hline E6 & 40 & 60 & 60 \\
\hline E7 & 60 & 70 & 60 \\
\hline E8 & 70 & 80 & 75 \\
\hline E9 & 45 & 55 & 60 \\
\hline E10 & 75 & 80 & 80 \\
\hline E11 & 70 & 75 & 80 \\
\hline E12 & 50 & 65 & 55 \\
\hline E13 & 65 & 60 & 65 \\
\hline E14 & 70 & 60 & 60 \\
\hline E15 & 75 & 75 & 80 \\
\hline
\end{tabular}

\section{QUADRO I}

RUN

ANÁLISE DE VARIÂNCIA A UM FACTOR

INPUT THE NO.OF TRIALS!3

INPUT THE NO.OF OBSERVATIONS

IN EACH TRIAL

!15

INPUT DATA ONE TRIAL AT A TIME

ERRORS ON INPUT CAN BE

CORRECTED AFTER EACH TRIAL

ENTRY

TRIAL 1

OBSERVATION $1=! 55$

OBSERVATION $2=! 60$

OBSERVATION $3=75$

OBSERVATION $4=! 65$

OBSERVATION $5=! 45$

OBSERVATION6 $=! 40$

OBSERVATION $7=! 60$

OBSERVATION $8=! 70$

OBSERVATION $9=! 45$

OBSERVATION $10=! 75$

OBSERVATION $11=! 70$

OBSERVATION $12=! 50$

OBSERVATION $13=! 65$

OBSERVATION $14=! 70$

OBSERVATION $15=! 75$

ARE DATA CORRECT(Y OR N)!Y

a Departamento de Química, Universidade de Coimbra. 
TRIAL 2

OBSERVATION $1=! 65$

OBSERVATION $2=! 65$

OBSERVATION $3=! 65$

OBSERVATION $4=! 70$

OBSERVATION $5=! 65$

OBSERVATION $6=! 60$

OBSERVATION $7=! 70$

OBSERVATION $8=! 80$

OBSERVATION $9=! 55$

OBSERVATION $10=! 80$

OBSERVATION $11=! 80$

OBSERVATION $12=! 55$

OBSERVATION $13=! 65$

OBSERVATION $14=! 60$

OBSERVATION $15=! 80$

ARE DATA CORRECT(Y OR N)! Y

TRIAL 3

OBSERVATION $1=50$

OBSERVATION $2=! 60$

OBSERVATION $3=! 60$

OBSERVATION $4=! 75$

OBSERVATION $5=! 70$

OBSERVATION $6=! 60$

OBSERVATION7 $=! 60$

OBSERVATION $8=! 75$

OBSERVATION $9=! 60$

OBSERVATION $10=! 80$

OBSERVATION $11=! 80$

OBSERVATION $12=! 55$

OBSERVATION $13=! 65$

OBSERVATION $14=! 60$

OBSERVATION $15=! 80$

SOURCE OF

VARIATION

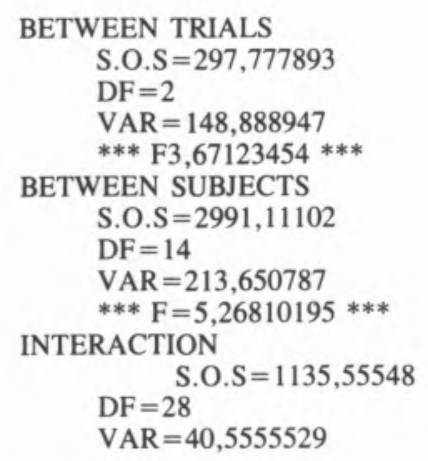

\section{Caso estudado II}

O estudo tem por finalidade verificar se a atitude dos estudantes em relação ao TL é afectada pelo uso de módulos de aprendizagem assistida por computador.

Os grupos e os tópicos envolvidos nas experiências usadas na simulação e no laboratório foram as seguintes:

GRUPO 1 Cinética da reacção do iodo com acetona GRUPO 2 Lei de Beer e determinação de concentrações GRUPO 3 Efeito da concentração no potencial de eléctrodo GRUPO 4 Determinação da energia de activação

Os Grupos 2 e 4 nas experiências simulada/laboratório e o Grupo 1 na experiência simulada utilizam um espectrofotómetro de absorção no visível. Os estudantes do Grupo
1 antes de realizarem as experiências no laboratório foram instruídos sobre o funcionamento do espectrofotómetro. Para estudos de correlação foi fornecido a cada estudante um teste escrito de resposta múltipla (4 opções) (Tabela 2). Os coeficientes de fidelidade dos testes foram determinados pelo método das metades. As metades foram organizadas a partir das somas das pontuações dos itens impares (variável 1) e a soma das pontuações dos itens pares (variável 2), calculando-se, posteriormente, a correlação entre as duas variáveis e, a seguir o coeficiente de fidelidade pela fórmula de Spearman-Brown $[3,4]$. Uma amostragem dos resultados obtidos por execução do programa "Coeficiente de correlação"' para a Experiência 2 relativa ao uso do espectrofotómetro, está indicada no Quadro II.

TABELA 2

Testes, número de questões $(N)$ e coeficientes de fidelidade

\begin{tabular}{lcc}
\hline Teste & $\mathrm{N}$ & $\begin{array}{c}\text { Coeficiente } \\
\text { de fidelidade }\end{array}$ \\
\hline Exp 1 & 14 & \\
Uso Esp. & 27 & 0,64 \\
Cinética & & 0,82 \\
Exp 2 & 17 & \\
Uso Esp. & 29 & 0,69 \\
Lei de Beer & 20 & 0,86 \\
Exp. 3 & 18 & 0,75 \\
Exp. 4 & & 0,91 \\
\hline
\end{tabular}

\section{QUADRO II}

RUN
COEFICIENTE DE CORRELAÇÃO

INPUT THE NO. OF OBS. (ROWS)

FOR EACH VARIABLE! 10

INPUT DATA ONE ROW AT A TIME

ERRORS ON INPUT CAN BE

CORRECTED AFTER 5 (OR ALL)

ROWS ARE ENTERED

ROW 1

VARIABLE $1=! 3$

VARIABLE $2=! 2$

ROW 2

VARIABLE $1=! 3$

VARIABLE $2=! 5$

ROW 3

VARIABLE $1=! 5$

VARIABLE $2=! 4$

ROW 4

VARIABLE $1=! 5$

VARIABLE $2=! 3$

ROW 5

VARIABLE $1=! 2$

VARIABLE $1=! 2$

ROW 6

VARIABLE $1=! 3$

VARIABLE $2=! 5$

ROW 7

VARIABLE $1=! 2$

VARIABLE $2=! 3$

ROW 8

VARIABLE $1=! 5$

ROW 9

VARIABLE $2=5$

VARIABLE $1=! 4$

VARIABLE $2=! 4$ 
ROW 10

VARIABLE $1=! 4$

VARIABLE $2=! 5$

ARE DATA CORRECT (Y OR N)!Y

CORRELATION COEFICIENT $=0,516129035$

COEFF. OF DETERMINATION $=0,26638918$

$\mathrm{N}=10$

DEGREES OF FREEDOM $=8$

A fidelidade para o teste com metade do número de itens é 0,52 . Aplicando a fórmula de Spearman-Brown, para a estimativa da fidelidade do teste com o dobro do número de itens, tem-se

$$
\mathrm{rn}(2)=2 \times 0,52 /(1+0,52)=0,68
$$

Referem-se de seguida os valores $\mathrm{F}$ gerados pelo programa "Análise de variância a dois factores"': factor A, atitude do estudante (atitude elevada $(\mathrm{H}) \times$ atitude baixa $(\mathrm{L})$ ), factor $B$, método de ensino utilizado (simulação $\times$ laboratório) e interacção (I) (Tabela 3). No Quadro III refere-se uma amostragem dos valores obtidos por execução do programa para o Teste 3.

\section{TABELA 3 \\ Valores $F$}

\begin{tabular}{ccccccc}
\hline Simulação & \multicolumn{3}{l}{ Laboratório } & & \multicolumn{2}{c}{ Valor F } \\
H & L & H & L & A & B & I
\end{tabular}

Exp 1

$\begin{array}{llllllll}\text { Cinética } & 11,66 & 9,54 & 10,83 & 8,95 & 8,73^{(3)} & 1,05 & 0,03\end{array}$

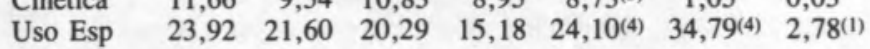

$\operatorname{Exp} 2$

$\lambda \max$

$\mathrm{Cu}-\lambda_{\max }$

$$
\begin{array}{lllllll}
12,00 & 8,21 & 10,07 & 6,92 & 22,13^{(4)} & 4,73^{(2)} & 0,19
\end{array}
$$

Exp 3

$\begin{array}{llllllll}\text { Teste } 3 & 19,90 & 16,17 & 20,11 & 14,54 & 42,48^{(4)} & 0,22 & 1,14\end{array}$

Exp 4

$\begin{array}{llllllll}\text { Teste } 4 & 18,14 & 14,14 & 12,90 & 7,50 & 6,38^{(2)} & 11,19^{(2)} & 0,14\end{array}$

(1) $\mathrm{p}<0.1$; (2) $\mathrm{p}<0,05 ;{ }^{(3)} \mathrm{p}<0,01 ;{ }^{(4)} \mathrm{p}<0,001$ [2]

\section{QUADRO III}

\section{RUN}

PROGRAMA ANÁLISE DE VARIÂNCIA A DOIS FACTORES

\section{ARRANGE DATA INTO A MATRIX}

WITH FACTOR 1 LEVELS IN ROWS

AND FACTOR 2 LEVELS IN COLS

INPUT NO.OF LEVELS IN

FACTOR $1 ! 2$

INPUT NO.OF LEVELS IN

FACTOR 2 ! 2

INPUT NO.OF OBS. IN EACH EACH

SAMPLE (CEL)!4

INPUT DATA.ERRORS ON INPUT

CAN BE CORRECTED AFTER EACH

SAMPLE INPUT
FACTOR 1,LEVEL(ROW) 1

FACTOR 2,LEVEL(COLUMN) 1

OBSERVATION $1=! 10.0$

OBSERVATION $2=! 11.1$

OBSERVATION $3=! 13.3$

OBSERVATION $4=! 12.2$

ARE DATA CORRECT(Y OR N)!Y

FACTOR 1,LEVEL(ROW) 1

FACTOR 2,LEVEL(COLUMN)2

OBSERVATION $1=! 9.7$

OBSERVATION $2=! 9.7$

OBSERVATION $3=! 10.8$

OBSERVATION $4=! 13.0$

ARE DATA CORRECT(Y OR N)!Y

FACTOR 1,LEVEL(ROW) 2

FACTOR 2,LEVEL(COLUMN)1

OBSERVATION $1=! 7.2$

OBSERVATION $2=! 7.5$

OBSERVATION $3=! 7.7$

OBSERVATION $4=! 7.9$

ARE DATA CORRECT(Y OR N)!Y

FACTOR 1,LEVEL(ROW) 2

FACTOR 2,LEVEL(COLUMN)2

OBSERVATION $1=! 8.1$

OBSERVATION $2=! 7.8$

OBSERVATION $3=! 8.0$

OBSERVATION $4=! 7.8$

ARE DATA CORRECT(Y OR N)!Y

\section{SOURCE OF VARIATION}

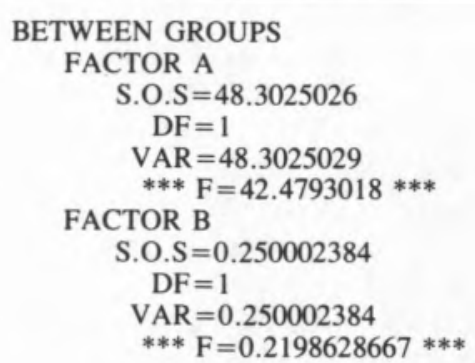

INTERACTION

S.O.S $=1.43999767$

$\mathrm{DF}=1$

VAR $=1.43999767$

$* * * \mathrm{~F}=1.26639599 * * *$

WITHIN GROUPS

S.O.S $=13.644999$

$\mathrm{DF}=12$

$\mathrm{VAR}=1.13708325$

O estudo mostra que o nível de performance atingido pelos estudantes dos grupos de simulação iguala $\mathrm{o}$ atingido pelos grupos laboratoriais. Atente-se, contudo, que as experiências seleccionadas para a simulação utilizam um instrumento muito específico e os resultados obtidos são expressos apenas em números. Não é pois de admirar, que para este tipo de experiência o nível de sucesso obtido seja superior ao alcançado em outro tipo de experiências que envolvem observações mais rigorosas ou uma elevada destreza manual. A utilidade da experimentação simulada neste tipo de experiências é reforçada pelo facto de não haver diferenças significativas entre as classificações obtidas nos testes práticos, quer pelos grupos de simulação quer pelos de laboratório, embora estes últimos evidenciem uma maior capacidade de destreza. Foi também evidenciada a utilidade da simulação como suplemento do laboratório e não como uma actividade extra. Os valores $\mathrm{H}$ são em todos os 
testes significativamente melhores, o que está de acordo com estudos realizados por outros investigadores no mesmo domínio [5,6]. Facto a salientar é o apoio que as experiências simuladas dão aos estudantes que trabalham no laboratório em ritmo individual. Neste caso, dado que não há limite de tempo, a percepção por parte do estudante do seu nível de sucesso é adiada para o fim da recolha e análise de dados, e do relato de resultados. Note-se ainda para os estudantes pertencentes à categoria atitude elevada, há uma tendência para serem mais cuidadosos e repetirem a experiência ou cálculos mais vezes, a fim de obterem uma maior segurança nos resultados. Os módulos de simulação, ao facilitarem o conhecimento imediato dos resultados, permi- tem aos estudantes progredirem na experiência mais rapidamente.

\section{Referências}

[1] A. Correia Cardoso, Maria Graça Miguel e S.J. Formosinho, Bol. Soc. Port. Química, 32/33, Junho/Setembro, 1988

[2] Pearson, E.S. e Hertley, H.O., (ed.): Biometrika Table for Statisticians, Cambridge Univ. Press, 1966

[3] Guilford, J.P., Psychometric Methods (2nd edition), New York, McGrawHill, 1954

[4] Gulliksen, H., Theory of Mental Tests, New York, 1950

[5] Porter, M.R. e Anderson, K.E., Science Education, 1969, 43, 12-19

[6] Reid, J.B., Palmer, R.I., Whitlock J., e Jones, J., Journal of Educational Psychology, 1973, 65, 65-73

\section{Convite à Reflexão...}

\section{As Virtudes da Metafísica: a química do átomo}

O carácter metafísico da teoria corpuscular, antes de Avogrado pelo menos, é evidente. Não havia possibilidade de a refutar. Podia-se sempre explicar o facto de não se conseguirem detectar os corpúsculos, nem qualquer prova deles, afirmando que eles eram demasiadamente pequenos para serem detectados. Só com uma teoria que levou a um cálculo do tamanho das moléculas é que se bloqueou mais ou menos esta via de fuga, de tal maneira que a refutação passou, em princípio, a ser possível. (As «verificaçōes» eram, em princípio, possíveis antes disso: a invenção do microscópio, digamos, poderia, imaginariamente, ter levado à descoberta de moléculas microscopicamente visíveis). Assim, o atomismo passou a ser testável logo que foi sujeito a um cálculo do tamanho de uma molécula. Este exemplo mostra que uma teoria não testável - uma teoria metafísica - se pode desenvolver e fortalecer até que se torne testável. Mas, a isto ser assim, parece grosseiramente enganador descrevê-la como não tendo significado.

[...] $\mathrm{O}$ atomismo primitivo era um sistema metafísico não só no sentido em que não era testável, mas também no sentido em que concebia o mundo em termos de uma vasta generalização, à maior escala possível: "não há mais nada senão os átomos e o vazio" (Leucipo, Demócrito). Os seus dois conceitos fundamentais, átomos e vazio, eram inobserváveis e, portanto, desconhecidos - como Demócrito salientou com uma lógica devastadora. Assim, o atomismo explicava o conhecido através do desconhecido: construía um mundo desconhecido e invisível por detrás do nosso mundo conhecido. E, precisamente por esta razão, foi consistentemente atacado por positivistas (mesmo depois de 1905), por todos os indutivistas de Bacon a Mach, e por todos os instrumentalistas de Barkeley a Duhem. A partir de 1905, os positivistas passaram a ser compreensivelmente mais reticentes sobre essa questão. Mas nunca explicaram como é que pode acontecer que um palanfrório sem significado possa ser substanciado em sentido. De facto, o exemplo do atomismo estabelece a inadequação da doutrina de que a metafísica é um mero palanfrório sem significado. Estabelece a inadequação da política de fazer pequenas mudanças subreptícias aqui e ali na doutrina de ausência de significado, com a esperança vã de a salvar.

Seja como for, o atomismo é um excelente exemplo de uma teoria não testável cuja influência sobre a ciência ultrapassou a de muitas teorias científicas testáveis.

Karl Popper, "O Realismo e o Objectivo da Ciência"
8

O 15,999

9

F

18,998
OXIGÉNIO, de oxys egenos, ou gerador de ácidos; descoberto em 1774; é o elemento mais abundante, constituindo cerca de metade do que existe na crosta terrestre, $21 \%$ da atmosfera, em volume, e $2 / 3$ do corpo humano. É respirado pelos animais e restituído à atmosfera pelas plantas.

FLUOR, de fluo, ou fluir; descoberto em 1771. O fluor é o mais reactivo dos não metais; apenas alguns gases nobres lhe resistem. Corroi a platina, material que resiste à maioria das outras substâncias químicas. Numa corrente de fluor gasoso, a madeira e a borracha inflamam-se e mesmo o amianto incandesce.

NEON, de neos, ou novo; descoberto em 1898. É o mais conhecido dos gases inertes e é usado principalmente em reclames. A lâmpada de neon é um tubo de vidro ao qual se fez o vácuo e introduziu uma quantidade muito pequena de neon gasoso: quando passa uma corrente eléctrica produz uma luz vermelha alaranjada muito brilhante. 